\title{
Does immunotherapy increase the rate of radiation necrosis after radiosurgical treatment of brain metastases?
}

\author{
${ }^{*}$ Rovel J. Colaco, MD, FRCR, ${ }^{1}$ Pierre Martin, BSc, ${ }^{2}$ Harriet M. Kluger, MD, ${ }^{3}$ James B. Yu, MD, and \\ Veronica L. Chiang, MD²
}

Departments of ${ }^{1}$ Therapeutic Radiology, ${ }^{2}$ Neurosurgery, and ${ }^{3}$ Medicine, Yale New Haven Hospital and Yale University School of Medicine, New Haven, Connecticut

\begin{abstract}
OBJECTIVE Radiation necrosis (RN), or its imaging equivalent, treatment-related imaging changes (TRIC), is an inflammatory reaction to high-dose radiation in the brain. The authors sought to investigate the hypothesis that immunotherapy increases the risk of developing RN/TRIC after stereotactic Gamma Knife (GK) radiosurgery for brain metastases.
\end{abstract}

METHODS A total of 180 patients who underwent GK surgery for brain metastases between 2006 and 2012 were studied. The systemic therapy they received was classified as cytotoxic chemotherapy (CT), targeted therapy (TT), or immunotherapy (IT). The timing of systemic therapy in relation to GK treatment was also recorded. Logistic regression was used to calculate the odds of developing $\mathrm{RN}$ according to type of systemic therapy received.

RESULTS The median follow-up time was 11.7 months. Of 180 patients, 39 (21.7\%) developed RN/TRIC. RN/TRIC rates were $37.5 \%$ (12 of 32) in patients who received IT alone, 16.9\% (14 of 83) in those who received CT only, and $25.0 \%$ (5 of 20 ) in those who received TT only. Median overall survival was significantly longer in patients who developed RN/TRIC (23.7 vs 9.9 months, respectively). The RN/TRIC rate was increased significantly in patients who received IT alone (OR $2.40[95 \% \mathrm{Cl} 1.06-5.44] ; p=0.03$ ), whereas receipt of any CT was associated with a lower risk of RN/TRIC (OR 0.38 [95\% Cl 0.18-0.78]; $p=0.01$ ). The timing of development of RN/TRIC was not different between patients who received IT and those who received CT.

CONCLUSIONS Patients who receive IT alone may have an increased rate of RN/TRIC compared with those who receive $\mathrm{CT}$ or TT alone after stereotactic radiosurgery, whereas receiving any CT may in fact be protective against RN/ TRIC. As the use of immunotherapies increases, the rate of RN/TRIC may be expected to increase compared with rates in the chemotherapy era.

http://thejns.org/doi/abs/10.3171/2015.6.JNS142763

KEY WORDS immunotherapy; radiation necrosis; stereotactic radiosurgery; brain metastases; oncology

$\mathrm{T}$ HE incidence of brain metastasis (BM) in adult patients with cancer has been estimated at up to $40 \%{ }^{1}$ Eighty percent of these patients will have multifocal disease,,$^{10}$ and $10 \%-15 \%$ of these metastases will be located in deep-seated inoperable regions of the CNS. ${ }^{21}$ With improvements in MRI-based screening and detection, as well as better overall survival (OS) through advances in radiotherapy, systemic chemotherapy, and new targeted (TT) and immunotherapy (IT) agents, the management of
BMs and the complications associated with their treatment are becoming increasingly important.

Stereotactic radiosurgery (SRS) is a popular alternative to whole-brain radiation therapy (WBRT) for the management of BMs, particularly because it is thought to be associated with fewer adverse neurocognitive effects than WBRT. ${ }^{2}$ SRS delivers higher doses of radiation than conventional fractionated radiotherapy in a highly conformal fashion to small volumes with a steep dose falloff. Cerebral

ABBREVIATIONS BM = brain metastases; $C$ CT = concurrent chemotherapy; $C T$ = cytotoxic chemotherapy; $G K=$ Gamma Knife; IT = immunotherapy; KPS = Karnofsky Performance Scale; OS = overall survival; RN = radiation necrosis; $\mathrm{SRS}$ = stereotactic radiosurgery; TRIC = treatment-related imaging changes; $\mathrm{TT}$ = targeted therapy; WBRT = whole-brain radiation therapy.

SUBMITTED December 17, 2014. ACCEPTED June 3, 2015.

INCLUDE WHEN CITING Published online November 6, 2015; DOI: 10.3171/2015.6.JNS142763.

${ }^{*}$ Dr. Colaco and Mr. Martin contributed equally to this work. 
radiation necrosis (RN) is the development of irreversible tissue injury in previously irradiated brain tissue that occurs more than 12 weeks after irradiation; $85 \%$ of cases present within 2 years after SRS. ${ }^{16}$ The incidence of RN has been estimated at up to $24 \%{ }^{4,11}$ Therefore, as patients with BMs survive longer because of advances in treatment, identifying patients at risk for $\mathrm{RN}$, differentiating $\mathrm{RN}$ and other treatment-related imaging changes (TRIC) from tumor progression, and managing TRIC, particularly in symptomatic patients, will likely become increasingly problematic.

Anecdotally, based on our institutional experience, there has been a rise in the incidence of RN/TRIC in patients undergoing SRS for BMs, the timing of which seems to correspond to the increase in use of IT at our institution in managing metastatic disease. Although this increase might be related solely to an increased rate of imaging surveillance after SRS in a population with increased survival, early reports ${ }^{5,9}$ have suggested that the use of some newer systemic IT and targeted therapy (TT) agents may also play a role. We therefore sought to investigate the hypothesis that treatment with IT might be a risk factor for developing RN/TRIC after SRS for BM.

\section{Methods}

This study was approved by the Yale University School of Medicine institutional review board. The medical records of 300 consecutively treated patients who underwent SRS with Gamma Knife (GK) at our institution (Yale New Haven Hospital) between 2006 and 2012 were retrospectively reviewed. All study patients required a histological diagnosis of malignancy and were required to have survived greater than 6 months after SRS with follow-up imaging available. A total of 189 patients survived more than 6 months, 182 of whom also had adequate follow-up imaging available. Two patients were found to have developed $\mathrm{RN}$ in lesions that had undergone treatment with GK twice and therefore were also excluded. A total of 180 patients were eligible for analysis. Patient demographics and tumor characteristics are detailed in Table 1.

\section{Radiotherapy}

All patients were treated using the Leksell Perfexion Gamma Knife (Elekta Medical Systems, Inc.). A planning MRI scan was obtained on the morning of GK treatment after application of a stereotactic head frame. Patients were scanned on a 1.5-T Siemens MRI scanner. Doublecontrast Gd was administered in every case unless the patient had significant renal dysfunction or contrast allergy, and $2.5-\mathrm{mm}$ slices were obtained for planning purposes. The images were transferred to the Gamma Plan version 10.1 software system (Elekta Instruments) for the purposes of treatment planning. The target volume was defined as the contrast-enhancing tumor volume, and dose selection was in accordance with RTOG (Radiation Therapy Oncology Group) 90-05 study guidelines. ${ }^{15}$

\section{Systemic Therapy}

The medical records of all patients were reviewed, and details of systemic therapy they received, including therapy before SRS, during SRS, less than 6 months after SRS,
TABLE 1. Baseline patient demographics

\begin{tabular}{lrc}
\hline Characteristic & No. of Patients & \% of Patients \\
\hline Sex & & \\
\hline Male & 80 & 44.4 \\
\hline Female & 100 & 55.6 \\
\hline Previous WBRT & & \\
\hline Yes & 55 & 30.5 \\
\hline No & 125 & 69.5 \\
\hline KPS score & & \\
\hline $80-100$ & 139 & 77.2 \\
\hline 70 & 15 & 8.3 \\
\hline $40-60$ & 10 & 5.6 \\
\hline Unknown & 16 & 8.9 \\
\hline Tumor histology & & \\
\hline Melanoma & 56 & 31.1 \\
\hline Lung & 71 & 39.4 \\
\hline Renal cell & 16 & 8.9 \\
\hline Breast & 27 & 15.0 \\
\hline Colorectal & 7 & 3.9 \\
\hline Other & 3 & 1.7 \\
\hline No. of BMs treated & & \\
\hline $1-4$ & 89 & 49.4 \\
\hline $5-9$ & 32 & 17.8 \\
\hline$\geq 10$ & 59 & \\
\hline
\end{tabular}

and more than 6 months after SRS, were recorded. Systemic agents were categorized as 1 of 3 types (Table 2): "traditional" cytotoxic chemotherapies (CTs), such as cisplatin; agents with a specific cell-signaling small-molecule target (targeted therapy; TT), such as erlotinib; or agents with a primary method of anticancer action that is through modulation of the immune system (immunotherapy; IT), such as ipilimumab (anti-CTLA-4). All patients included in the study were receiving ongoing systemic therapy for the presence of persistent systemic disease.

\section{Follow-Up Evaluation}

Patients were evaluated 6 weeks after GK surgery and at 3-month intervals thereafter with both clinical and MRI follow-up. Patients with lesions suspicious for RN/ TRIC were discussed at the tumor board, and a management plan was formulated. Patients were identified in this study as having RN/TRIC if they had either histologically confirmed RN or a lesion that increased in size on Gdenhanced T1-weighted MRI associated with increasing FLAIR signal after SRS followed by spontaneous resolution on subsequent serial scans (Fig. 1). In the latter case, when possible, $\left[{ }^{18} \mathrm{~F}\right] \mathrm{FDG}$ PET scans and/or MRI spectroscopy studies were also used to confirm or exclude tumor recurrence.

\section{Statistical Analysis}

The primary end point was the development of either tissue-confirmed $\mathrm{RN}$ or radiological changes supportive of RN/TRIC. Time to development of RN/TRIC was 
TABLE 2. Systemic therapies according to category

\begin{tabular}{lll}
\hline \multicolumn{1}{c}{ CT } & \multicolumn{1}{c}{ TT } & \multicolumn{1}{c}{ IT } \\
\hline 5-Fluorouracil & Bevacizumab & Anti-CD 137 \\
\hline Abraxane & Cetuximab & Anti-CTLA-4 \\
\hline Adriamycin & Dabrafenib & Anti-PD-1 \\
\hline Capecitabine & Dasatinib & Interferon \\
\hline Carboplatin & Erlotinib & Interleukin 2 \\
\hline Cisplatin & Everolimus & \\
\hline Cyclophosphamide & Gefitinib & \\
\hline Dacarbazine & Herceptin & \\
\hline Etoposide & Lapatinib & \\
\hline Gemcitabine & Letrozole & \\
\hline Ifosfamide & Panitumumab & \\
\hline Ixabepilone & Sorafenib & \\
\hline Methotrexate & Sunitinib & \\
\hline Mitomycin C & Tamoxifen & \\
\hline Navelbine & Temsirolimus & \\
\hline Oxaliplatin & Vemurafenib & \\
\hline Paclitaxel & \\
\hline Pemetrexed & \\
\hline Temozolomide & \\
\hline Vinblastine & \\
\hline
\end{tabular}

measured from the day of GK treatment of the lesion that developed RN/TRIC. For lesions in which RN was diagnosed by imaging alone, the date of the MRI/PET scan at which RN/TRIC was first suspected was taken as the date at which the primary end point was reached. For histologically confirmed RN, the date of the final pathology report was the date of the primary end point. Univariate and multivariate logistic regression was performed to assess the impact of type of systemic therapy on the risk of developing RN/TRIC.

\section{Results}

\section{Patient Demographics and Treatment}

The median follow-up time was 11.7 months (range
6-48 months). Baseline patient characteristics are shown in Table 1. Seventy-one patients (39.4\%) had a primary diagnosis of lung cancer, 56 (31.1\%) had melanoma, 27 $(15.0 \%)$ had breast cancer, $16(8.9 \%)$ had renal cancer, 7 (3.9\%) had colorectal cancer, and 3 (1.7\%) had another type of cancer. Fifty-five (30.5\%) patients had undergone WBRT before SRS, and 154 (85.6\%) had a baseline Karnofsky Performance Scale (KPS) score of $\geq 70$. The median GK prescription dose for each lesion was $20 \mathrm{~Gy}$ (range $15-24 \mathrm{~Gy}$ ) to the $50 \%$ isodose surface.

The distribution of patients who received CT, IT, or TT either alone or in combination is shown in Table 3. Of 180 patients total, 83 were treated with chemotherapy alone, 32 with IT alone, and 20 with TT only. Four patients did not receive any systemic therapy, and the remaining 41 patients received a combination of therapies over the course of their follow-up. For the purposes of multivariate analysis, 42 patients were identified as having received any IT during their treatment course, either alone $(\mathrm{n}=32)$ or in combination with CT $(n=4)$, TT $(n=4)$, or both TT and $\mathrm{CT}(\mathrm{n}=2)$.

\section{Outcomes}

Of the 180 study patients, 39 developed RN/TRIC as defined by our criteria. One hundred thirty-five had stability or regression of their treated lesions (defined here as stable disease), and 6 patients had histologically documented tumor recurrence.

The median OS for the 180 patients was 11.8 months (95\% CI 10.0-15.2 months). For patients with RN/TRIC, the median OS was significantly longer for those with RN/ TRIC at 23.7 months (95\% CI 18.3-38.5 months) than for those in the group without RN/TRIC at 9.9 months $(95 \%$ CI 7.9-11.7 months) $(\mathrm{p}=0.01)$.

Of the 39 patients with RN/TRIC, 18 were symptomatic, including 11 patients who underwent surgical intervention and 7 who were diagnosed on imaging and managed with steroids only. The median time to development of RN in asymptomatic patients was 8.7 months (range 3.030.0 months), whereas in symptomatic patients, it was 7.5 months (range 3.0-62.3 months). Tumor volumes treated were not significantly different between the lesions that developed RN/TRIC versus those that did not (mean volume


FIG. 1. A: Treatment-planning MR image showing the lesion treated with SRS on the day of GK treatment. B: MR image showing an initial increase in lesion size 6 months after GK surgery. C: MR image showing spontaneous resolution/reduction in size of the previously enlarging lesion 9 months after GK surgery. 
TABLE 3. Rates of RN according to systemic therapy type

\begin{tabular}{|c|c|c|c|}
\hline Systemic Therapy & $\begin{array}{c}\text { Total No. of } \\
\text { Patients }(n=180)\end{array}$ & Total w/ RN $(n=39)$ & $\%$ \\
\hline IT only & 32 & 12 & 37.5 \\
\hline TT only & 20 & 5 & 25.0 \\
\hline CT only & 83 & 14 & 16.9 \\
\hline $\mathrm{IT}+\mathrm{TT}$ & 4 & 2 & 50.0 \\
\hline $\mathrm{IT}+\mathrm{CT}$ & 4 & 0 & 0.0 \\
\hline $\mathrm{TT}+\mathrm{CT}$ & 31 & 5 & 16.1 \\
\hline $\mathrm{IT}+\mathrm{TT}+\mathrm{CT}$ & 2 & 0 & 0.0 \\
\hline None & 4 & 1 & 25.0 \\
\hline
\end{tabular}

of RN/TRIC lesions $3.89 \mathrm{~cm}^{3}$ [range $0.02-17.3 \mathrm{~cm}^{3}$ ] vs 7.13 $\mathrm{cm}^{3}$ [range $0.006-187.4 \mathrm{~cm}^{3}$ ]). Treatment doses were also not significantly different between the lesions that developed RN/TRIC versus those that did not (median prescription dose for RN/TRIC lesions 20 Gy [range 16-24 Gy] vs 18 Gy [range 15-24 Gy]).

For patients who received any IT, the median OS was 9.3 months (95\% CI 7.3-18.5 months) compared with 10.0 months (95\% CI 10.6-11.7 months) for patients who received no IT at all. Patients with RN/TRIC who received IT alone had a median OS of 23.7 months (95\% CI 13.8 months-[upper limit not reached]), whereas for patients with RN/TRIC who received no IT, the median OS was 22.7 months $(95 \%$ CI 14.5 months-[upper limit not reached]).

The median OS for patients who received IT, TT, or CT alone who developed RN/TRIC were 23.7 months (95\% CI 13.8 months-[upper limit not reached]), 26.2 months (95\% CI 12.7 months-[upper limit not reached]), and 14.5 months (95\% CI 10.0 months-[upper limit not reached]), respectively, compared with 9.3 months (95\% CI 6.0-34.2 months), 31.0 months (95\% CI 7.5-63.6 months), and 10.0 months (95\% CI 7.4-12.6 months), respectively, for those who received IT, TT, or CT alone who did develop RN/ TRIC.

\section{Systemic Therapy and Rates of RN/TRIC}

Twelve (37.5\%) of the 32 patients who received IT alone developed RN/TRIC (Table 3). Five of the 20 patients who were treated with TT alone developed RN/TRIC (25.0\%), and $14(16.9 \%)$ of the 83 patients who received CT alone developed RN/TRIC. One (25\%) of the 4 patients who did not receive any systemic therapy also developed RN.

The results of univariate and multivariate analysis according to RN group are shown in Table 4 .

Receiving IT alone was associated with a significantly increased risk of RN/TRIC on univariate analysis (OR 2.40 [95\% CI 1.06-5.44]; $\mathrm{p}=0.03$ ), with a trend toward statistical significance on multivariate analysis (OR 2.71 [95\% CI 0.94-7.76]; $\mathrm{p}=0.06$ ); however, TT alone and CT alone were not statistically significant on univariate or multivariate analysis.

For patients who received any IT as part of their systemic treatment (either alone or in combination with TT and/or CT), there was a trend in univariate analysis toward an increased risk of RN/TRIC (OR 2.07 [95\% CI 0.964.47]; $p=0.06$ ), although this trend was not statistically significant, and the effect was lost on multivariate analysis (OR 0.98 [95\% CI 0.31-3.11]; $\mathrm{p}=0.97$ ).

Receiving any CT (either alone or in combination with TT and/or IT), however, was found to be significantly associated with a decreased risk of RN/TRIC on univariate analysis (OR 0.38 [95\% CI 0.18-0.78]; $\mathrm{p}=0.01$ ), with a trend toward statistical significance on multivariate analysis (OR 0.38 [95\% CI 0.13-1.06]; $\mathrm{p}=0.06$ ). No other demographic or treatment factors, including KPS score, tumor histology, previous WBRT, tumor volumes, or treatment dose, were found to be statistically significantly associated with RN/TRIC on univariate or multivariate analyses.

\section{Time to Development of RN/TRIC}

The median time to development of RN/TRIC was 9.5 months overall (range 1.7-63.3 months). There was no significant difference in time to $\mathrm{RN}$ according to systemic therapy type $(p=0.50)$. For patients who received any IT, the median time to development of RN was 10.2 months (range 2.8-22.1 months), whereas the time to RN for those who received IT only was also 10.2 months (range 2.822.1 months). For patients who did not receive any IT, the median time to development of $\mathrm{RN}$ was 8.7 months (range 1.7-63.3 months). No significant relationship was found between the timing of use of IT/TT or CT and the timing of GK administration.

\section{Discussion}

Radiation necrosis is an inflammatory response to irreversible tissue injury that develops in sites of high-dose radiation. ${ }^{19}$ Sheline et al ${ }^{16}$ originally described cerebral $\mathrm{RN}$ as irreversible tissue injury that occurs greater than 12 weeks after cranial irradiation. More recently, this entity, specifically occurring after radiosurgery, was characterized histologically as a central area of necrosis surrounded by regions of vascular hyalinization with or without vasculitis, demyelination, macrophage and T-cell infiltration, and reactive astrocytosis. ${ }^{13,20}$

Estimates of the incidence of symptomatic RN vary between $7 \%$ and $14 \%$; when combined with asymptomatic $\mathrm{RN}$, the incidence has been estimated at up to $24 \%$ and may even be higher, because patients with BMs are living longer today than previously reported..$^{4,11}$ The true incidence of RN remains unknown for a number of reasons. First, there is no single test for RN. Although the gold standard remains biopsy and histological examination of a suspected lesion, patients are frequently not fit enough or are unwilling to undergo surgery, and even if tissue is obtained, it may be subject to sampling error. Second, RN is notoriously difficult to differentiate from tumor progression by imaging. There is still no consensus regarding the optimum imaging techniques and characteristics that confer a diagnosis of RN. For this reason, we decided to apply the nomenclature of TRIC in our study for lesions that clinically behaved like RN but were not confirmed by histology.

Efforts to identify risk factors associated with the de- 
TABLE 4. Univariate and multivariate analysis of risk of developing RN

\begin{tabular}{lccccc}
\hline \multirow{2}{*}{ Risk Factor } & \multicolumn{2}{c}{ Univariate Analysis } & & \multicolumn{2}{c}{ Multivariate Analysis } \\
\cline { 2 - 3 } \cline { 5 - 6 } & OR $(95 \% \mathrm{Cl})$ & $\mathrm{p} \mathrm{Value}$ & & OR $(95 \% \mathrm{Cl})$ & $\mathrm{p} \mathrm{Value}$ \\
\hline Age & $0.97(0.94-1.00)$ & 0.06 & $0.97(0.94-1.00)$ & 0.08 \\
\hline Sex (male vs female) & $0.96(0.47-1.96)$ & 0.90 & $\mathrm{NA}$ & - \\
\hline Histology (melanoma vs other) & $0.84(0.38-1.83)$ & 0.66 & $0.38(0.13-1.06)$ & 0.06 \\
\hline Any CT & $0.38(0.18-0.78)$ & 0.01 & $0.84(0.34-2.10)$ & 0.71 \\
\hline Any TT & $1.04(0.49-2.26)$ & 0.12 & $0.98(0.31-3.11)$ & 0.97 \\
\hline Any IT & $2.07(0.96-4.47)$ & 0.06 & $1.10(0.41-2.95)$ & 0.84 \\
\hline CT only & $0.58(0.28-1.21)$ & 0.15 & $1.96(0.53-7.24)$ & 0.31 \\
\hline TT only & $1.44(0.48-4.34)$ & 0.51 & $2.71(0.94-7.76)$ & 0.06 \\
\hline IT only & $2.40(1.06-5.44)$ & 0.03 & NA & - \\
\hline Previous WBRT (yes vs no) & $0.79(0.25-1.76)$ & 0.56 & & & - \\
\hline
\end{tabular}

$\mathrm{NA}=$ not included in the multivariate analysis.

velopment of RN have focused primarily on dose and irradiated volumes rather than other factors such as systemic therapy. Kjellberg and $\mathrm{Abe}^{7}$ created a dose-versus-volume model that was thought to predict a $1 \%$ risk of $\mathrm{RN}$, whereas Flickinger et al. ${ }^{6}$ found that a $12-G y$ dose volume was predictive for RN in patients treated with SRS for arteriovenous malformations. In a study of 426 patients with glioma, Ruben et al..$^{14}$ found that adjuvant chemotherapy, age, total dose, fraction size, and chemotherapy were all associated with an increased risk of RN. With regards to BM, RTOG 90-05 established safe guidelines for singlefraction SRS in the treatment of BM and demonstrated the link between increasing radiation dose per fraction and an increased risk of RN. ${ }^{15}$ To date, however, no known study has examined the effect of systemic treatment (and more specifically IT and TTs) on the risk of developing RN in the treatment of BM either with or without SRS.

A recent preliminary report by Du Four et al. ${ }^{5}$ of 3 patients who received ipilimumab after SRS for metastatic melanoma and developed RN acknowledged the importance of considering the impact of such therapies on RN, although the authors were unable to conclude whether ipilimumab might increase the risk of developing RN. Liebner et al. ${ }^{9}$ also recently reported 2 cases of rapid development of RN after SRS with the administration of TT using the BRAF inhibitor vemurafenib 3 or 4 months after SRS. In both cases, the lesions were resected completely, and pathology revealed RN only with no viable tumor. Several recent studies, published in abstract form, evaluated risk factors for RN in patients with BM who received SRS. , $^{8,17,18}$ Kohutek et al. ${ }^{8}$ investigated clinical factors associated with $\mathrm{RN}$ in 118 patients with BM, including chemotherapy and bevacizumab within 8 weeks of SRS. On multivariate analysis, only tumor size, previous WBRT, and KPS score were associated with RN. The results of our study suggest that IT is a factor that may contribute independently to the development of RN/TRIC. The mechanism by which IT may increase RN/TRIC is not known, but in theory, any inflammatory response of the brain to high-dose radiation could be exacerbated by immune response potentiators. What may confound the clinical picture in some cases is the reluctance to use steroids when a patient is receiving active IT treatment.

In a study by Sneed et al. ${ }^{18}$ of 436 patients with BM, the authors found that the risk of RN varied significantly according to tumor histology (1-year probability for RN with renal and lung cancer $15 \%-23 \%$ and $10 \%-16 \%$, respectively, compared with 6\%-9\% for melanoma and $3 \%-6 \%$ for breast cancer), BM diameter, and history of previous cranial irradiation. Their evaluation of concurrent chemotherapy (CCT) as a risk factor for $\mathrm{RN}$, however, is still ongoing. More recently, Shiue et al. ${ }^{17}$ reported on 334 patients with BM in whom a number of risk factors were examined for their effect on the risk of developing $\mathrm{RN}$, including CCT and TT. It is interesting to note that the authors reported that CCT was thought to reduce the risk of RN. The mechanism by which chemotherapy may suppress RN/TRIC is not known. One hypothesis that may support the observation of Shiue et al. ${ }^{17}$ with regards to CCT may be the cellular suppressive effect of chemotherapy, which may reduce the inflammatory response that causes RN. This theory is supported by the findings in our study, in which patients who received any CT as part of their systemic therapy were found to have a reduced risk of developing RN/TRIC.

The interaction of several different forms of systemic therapy may therefore act as a confounding factor in that if IT does indeed increase the risk of $\mathrm{RN}$ and chemotherapy acts as a protective factor, the degree to which these systemic therapies might offset each other with regards to the risk of developing $\mathrm{RN}$ is unknown and warrants prospective study.

The development of RN/TRIC may also be related to duration of survival. We previously reported that the development of TRIC is associated with improved survival. ${ }^{12}$ In this study, patients who developed RN/TRIC also had a much greater OS than those who did not develop RN/ TRIC (23.7 vs 9.9 months, respectively). It is interesting to note that this improved median OS was found regardless of which type of systemic therapy the patient received. Because RN/TRIC is a delayed treatment effect and can occur up to 2 years after SRS, ${ }^{16}$ it is possible that only 
those who responded to their systemic therapies will develop RN/TRIC, with the rate of response being higher in patients who receive IT than in their counterparts who receive CT. Last, an increasing incidence of RN/TRIC with IT might be simply a result of increased diagnostic awareness of RN coupled with extended survival in patients treated with IT/TT. Further prospective study is needed to examine this question, and a standard diagnostic and treatment algorithm for RN, such as that suggested by Chao et al., ${ }^{3}$ may also prove useful in this regard.

The findings in this study may be hampered by the disadvantages of a retrospective study, including that some systemic therapy records may have been incomplete and the lack of a single set of diagnostic criteria for RN/TRIC. Likely because of the small size of our study cohort, we could not elicit the impact of the timing of IT and/or chemotherapy relative to the timing of GK surgery in this study, and further prospective study is needed. Furthermore, given the different mechanisms of action of the different ITs lumped together in this study, it is possible that their interactions with GK surgery may not have been homogeneous. Future studies that concentrate on single mechanisms of action and their interactions with GK surgery are also needed. Last, we were not able to confirm the radiological findings of TRIC by specifically studying the immunological changes in resected RN specimens. A better understanding of the histological correlation to radiographic findings is much needed.

In summary, because neurosurgeons are called to salvage lesions regrowing radiographically after radiosurgery, it is important first to recognize that not all radiographic regrowth represents tumor regrowth; and second, it is important to recognize that RN/TRIC may be more possible in these cases if the patient is or was receiving IT treatment for the cancer. In addition, given the increased likelihood of developing RN/TRIC after radiosurgery, if immunotherapy is likely to be part of systemic therapy for a patient with single BM, the neurosurgeon may wish to reconsider whether resection (rather than SRS) might be the better treatment option for BM if the patient is a surgical candidate. The results of this study suggest that the rate of $\mathrm{RN} / \mathrm{TRIC}$ after radiosurgery for BM is higher in patients who receive IT than those who receive chemotherapy. Regardless of whether chemotherapy suppresses RN/TRIC or IT exacerbates it, with the increasing use of immunebased agents in cancers with a predilection for metastasizing to the brain, such as melanoma and lung, breast, colon, and renal cancer, the rate of cerebral RN/TRIC after SRS is likely to rise, and further studies to document its impact on patient outcomes are important. Study is needed to determine the exact mechanism, at the molecular level, through which IT might contribute to the development of RN/TRIC. In addition, the sequence of administration of these therapies in association with the timing of RN/TRIC development warrants further evaluation.

\section{Conclusions}

Patients who received IT alone have an increased rate of RN/TRIC over those who receive CT or TT alone. Receiving CT, either alone or in combination with IT and/or
TT, decreases the risk of developing RN/TRIC after SRS for BM. As the use of IT in patients with BM increases, an increase in the RN/TRIC rate may be expected after radiosurgical treatment.

\section{References}

1. Cairncross JG, Kim JH, Posner JB: Radiation therapy for brain metastases. Ann Neurol 7:529-541, 1980

2. Chang EL, Wefel JS, Hess KR, Allen PK, Lang FF, Kornguth DG, et al: Neurocognition in patients with brain metastases treated with radiosurgery or radiosurgery plus whole-brain irradiation: a randomised controlled trial. Lancet Oncol 10:1037-1044, 2009

3. Chao ST, Ahluwalia MS, Barnett GH, Stevens GH, Murphy ES, Stockham AL, et al: Challenges with the diagnosis and treatment of cerebral radiation necrosis. Int J Radiat Oncol Biol Phys 87:449-457, 2013

4. Chin LS, Ma L, DiBiase S: Radiation necrosis following gamma knife surgery: a case-controlled comparison of treatment parameters and long-term clinical follow up. J Neurosurg 94:899-904, 2001

5. Du Four S, Wilgenhof S, Duerinck J, Michotte A, Van Binst A, De Ridder M, et al: Radiation necrosis of the brain in melanoma patients successfully treated with ipilimumab, three case studies. Eur J Cancer 48:3045-3051, 2012

6. Flickinger JC, Kondziolka D, Lunsford LD, Kassam A, Phuong LK, Liscak R, et al: Development of a model to predict permanent symptomatic postradiosurgery injury for arteriovenous malformation patients. Int J Radiat Oncol Biol Phys 46:1143-1148, 2000

7. Kjellberg RN, Abe M: Stereotactic Bragg peak proton beam therapy, in Lunsford LD (ed): Modern Stereotactic Neurosurgery. Boston: Martinus Nijhoff, 1988, pp 463-470

8. Kohutek Z, Chan T, Yamada J, Zhang Z, Brennan C, Tabar V, et al: Clinical factors associated with radiation necrosis after stereotactic radiosurgery for brain metastases. Int J Radiat Oncol Biol Phys 84:S117, 2012

9. Liebner DA, Walston SA, Cavaliere R, Powers CJ, Sauvageau E, Lehman NL, et al: Radiation necrosis mimicking rapid intracranial progression of melanoma metastasis in two patients treated with vemurafenib. Melanoma Res 24:172-176, 2014

10. Lohr F, Pirzkall A, Hof H, Fleckenstein K, Debus J: Adjuvant treatment of brain metastases. Semin Surg Oncol 20:50-56, 2001

11. Minniti G, Clarke E, Lanzetta G, Osti MF, Trasimeni G, Bozzao A, et al: Stereotactic radiosurgery for brain metastases: analysis of outcome and risk of brain radionecrosis. Radiat Oncol 6:48, 2011

12. Patel TR, McHugh BJ, Bi WL, Minja FJ, Knisely JP, Chiang VL: A comprehensive review of MR imaging changes following radiosurgery to 500 brain metastases. AJNR Am J Neuroradiol 32:1885-1892, 2011

13. Rauch PJ, Park HS, Knisely JP, Chiang VL, Vortmeyer AO: Delayed radiation-induced vasculitic leukoencephalopathy. Int J Radiat Oncol Biol Phys 83:369-375, 2012

14. Ruben JD, Dally M, Bailey M, Smith R, McLean CA, Fedele $P$ : Cerebral radiation necrosis: incidence, outcomes, and risk factors with emphasis on radiation parameters and chemotherapy. Int J Radiat Oncol Biol Phys 65:499-508, 2006

15. Shaw E, Scott C, Souhami L, Dinapoli R, Kline R, Loeffler J, et al: Single dose radiosurgical treatment of recurrent previously irradiated primary brain tumors and brain metastases: final report of RTOG protocol 90-05. Int J Radiat Oncol Biol Phys 47:291-298, 2000

16. Sheline GE, Wara WM, Smith V: Therapeutic irradiation and brain injury. Int J Radiat Oncol Biol Phys 6:1215-1228, 1980

17. Shiue K, Barnett GH, Stockham A, Ahluwalia M, Suh JH, 
Reddy C, et al: Lack of response is a risk factor for radiation necrosis after radiosurgery for brain metastases. Int J Radiat Oncol Biol Phys 87:S278, 2013

18. Sneed PK, Mendez J, Fogh SE, Barani IJ, Ma L, McDermott MW: Risk factors for radiation necrosis after radiosurgery for brain metastases. Int J Rad Onc Biol Phys 84:S118-S119, 2012

19. Szeifert GT, Atteberry DS, Kondziolka D, Levivier M, Lunsford LD: Cerebral metastases pathology after radiosurgery: a multicenter study. Cancer 106:2672-2681, 2006

20. Szeifert GT, Kondziolka D, Levivier M, Lunsford LD: Histopathology of brain metastases after radiosurgery. Prog Neurol Surg 25:30-38, 2012

21. Walker AE, Robins M, Weinfeld FD: Epidemiology of brain tumors: the national survey of intracranial neoplasms. Neurology 35:219-226, 1985

\section{Disclosure}

The authors report no conflict of interest concerning the materi- als or methods used in this study or the findings specified in this paper.

\section{Author Contributions}

Conception and design: Chiang, Colaco, Yu. Acquisition of data: Chiang, Colaco, Martin. Analysis and interpretation of data: Chiang, Martin, Yu. Drafting the article: Chiang, Colaco, Martin, Yu. Critically revising the article: all authors. Reviewed submitted version of manuscript: Chiang, Martin, Kluger, Yu. Approved the final version of the manuscript on behalf of all authors: Chiang. Statistical analysis: Yu. Administrative/technical/material support: Kluger.

\section{Correspondence}

Veronica L. Chiang, Department of Neurosurgery, Yale University School of Medicine, 333 Cedar St., New Haven, CT 06520. email: veronica.chiang@yale.edu. 